\title{
Systematic Scoring Balloon Lesion Preparation for Drug-Coated Balloon Angioplasty in Clinical Routine: Results of the PASSWORD Observational Study
}

\author{
Klaus Bonaventura - Markus Schwefer - Ahmad Khairuddin Mohamed Yusof • \\ Matthias Waliszewski · Florian Krackhardt • Philip Steen • \\ Raymundo Ocaranza · Ahmad Syadi Zuhdi · Liew Houng Bang • \\ Kristof Graf · Ulrich Böck · Kenneth Chin
}

Received: February 24, 2020 / Published online: April 9, 2020

(c) The Author(s) 2020

\section{ABSTRACT}

Introduction: Scoring balloon angioplasty (SBA) for lumen gain prior to stent implantations or drug-coated balloon angioplasty (DCB) is considered an essential interventional tool for lesion preparation. Recent evidence indicates that SBA may play a pivotal role in enhancing the angiographic and clinical outcomes of DCB angioplasty. Methods: We studied the systematic use of SBA with a low profile, non-slip element device prior to DCB angioplasty in an unselected, non-

Digital Features To view digital features for this article go to https://doi.org/10.6084/m9.figshare.12018669.

K. Bonaventura $(\bowtie) \cdot P$. Steen

Klinikum Ernst Von Bergmann, Potsdam, Germany

e-mail: Klaus.Bonaventura@klinikumevb.de

M. Schwefer

Elblandklinikum Riesa, Riesa, Germany

A. K. M. Yusof

The National Heart Institute of Malaysia, Kuala

Lumpur, Malaysia

M. Waliszewski · P. Steen

Medical Scientific Affairs, B. Braun Melsungen AG,

Berlin, Germany

M. Waliszewski · F. Krackhardt

Department of Cardiology and Internal Medicine,

Charité - Universitätsmedizin Berlin, Campus

Virchow, Berlin, Germany randomized patient population. This prospective, all-comers study enrolled patients with de novo lesions as well as in-stent restenotic lesions in bare metal stents (BMS-ISR) and drug-eluting stents (DES-ISR). The primary endpoint was the target lesion failure (TLF) rate at 9 months (ClinicalTrials.gov Identifier NCT02554292).

Results: A total of 481 patients (496 lesions) were recruited to treat de novo lesions $(78.4 \%, 377)$, BMS-ISR $(4.0 \%, 19)$, and DES-ISR $(17.6 \%, 85)$. Overall risk factors were acute coronary syndrome (ACS, 20.6\%, 99), diabetes mellitus $(46.8 \%, 225)$, and atrial fibrillation $(8.5 \%, 41)$. Average lesion lengths were $16.7 \pm 10.4 \mathrm{~mm}$ in the de novo group, and $20.1 \pm 8.9 \mathrm{~mm}$ (BMS-ISR) and $16.2 \pm 9.8 \mathrm{~mm}$ (DES-ISR) in the ISR groups. Scoring

\section{R. Ocaranza}

Hospital Universitario Lucus Augusti, Lugo, Spain

A. S. Zuhdi

Pusat Perubatan Universiti Malaya, Kuala Lumpur, Malaysia

L. H. Bang

Hospital Queen Elizabeth II, Kota Kinabalu, Sabah, Malaysia

K. Graf

Jüdisches Krankenhaus Berlin, Berlin, Germany

U. Böck

Marien-Hospital Marl, Marl, Germany

K. Chin

Pantai Hospital Kuala Lumpur, Kuala Lumpur, Malaysia 
balloon diameters were $2.43 \pm 0.41 \mathrm{~mm}$ (de novo), $2.71 \pm 0.31 \mathrm{~mm}$ (BMS-ISR), and $2.92 \pm 0.42 \mathrm{~mm}$ (DES-ISR) whereas DCB diameters were $2.60 \pm 0.39 \mathrm{~mm}$ (de novo), $3.00 \pm 0.35 \mathrm{~mm}$ (BMSISR), and $3.10 \pm 0.43 \mathrm{~mm}$ (DES-ISR), respectively. The overall accumulated TLF rate of 3.0\% (14/463) was driven by significantly higher target lesion revascularization rates in the BMS-ISR $(5.3 \%, 1 / 19)$ and the DES-ISR group $(6.0 \%, 5 / 84)$. In de novo lesions, the TLF rate was $1.1 \%(4 / 360)$ without differences between calcified and non-calcified lesions $(p=0.158)$ and small vs. large reference vessel diameters with a cutoff value of $3.0 \mathrm{~mm}(p=0.901)$. Conclusions: The routine use of a non-slip element scoring balloon catheter to prepare lesions suitable for drug-coated balloon angioplasty is associated with high procedural success rates and low TLF rates in de novo lesions.

Keywords: De novo lesion; Drug-coated balloon; Lesion preparation; Scoring balloon; Stent-less intervention; Target lesion failure

\section{Key Summary Points}

Why carry out this study?

Scoring balloon angioplasty (SBA) for acute lumen gain is an interventional tool for lesion preparation

Non-slip element scoring balloon catheter may be associated with enhanced lesion crossability

Drug-coated balloon (DCB) angioplasty was recently studied in small vessel de novo artery disease in the BASKETSMALL II trial

\section{What was learned from the study?}

The combination of non-slip element scoring balloon catheter and DCB was safe and effective in the treatment of de novo coronary artery disease with target lesion failure rates of less than $2 \%$ at 9 months

There are no de novo subgroups, e.g., calcified lesions, larger vessels $(\geq 3 \mathrm{~mm})$ which do not benefit from SBA/DCB treatment

\section{INTRODUCTION}

Since the early days of percutaneous coronary interventions (PCI) the complexity of treated lesions has steadily increased. In parallel, the number of interventional tools to improve the procedural success rate has also expanded, leaving the interventionalist with a large array of options. A special focus is currently paid to lesion preparation, partly because heavily calcified and fibrotic lesions, which are not very amenable for sufficient luminal gain, continue to present an important challenge in coronary interventions.

In this context, a minimalistic approach appears to be especially intriguing. The concept of lesion preparation in challenging situations for subsequent stent implantation is not new and was even introduced prior to the stent era. Mauri et al. [1] reported the results from a randomized controlled trial comparing plain old balloon angioplasty (POBA) and cutting balloon angioplasty (CBA). They found that there were no angiographic improvements in the lesions treated with CBA as compared to POBA and concluded that CBA should be reserved for more complex lesion morphologies. Danek et al. [2] conducted a study-level meta-analysis by comparing available lesion modification strategies, which were CBA, scoring balloon angioplasty (SBA), and rotational and orbital atherectomy. They reported improved longterm outcomes in pre-stenting lesion modification as compared to balloon pre-dilatation or no pre-dilatation at all. This advantage, however, could only be observed in pooled data from randomized controlled trials.

Whereas the lesion modification strategies mentioned above were primarily studied in the context of luminal gain prior to stent implantation [3], renewed interest has been generated with the introduction of drug-coated balloon (DCB) angioplasty. Lumen gain prior to DCB angioplasty may be of paramount importance in the absence of stent or scaffold implantations due to elastic recoil and/or dissections. However, pivotal studies for DCB angioplasty were primarily conducted to treat in-stent restenotic (ISR) lesions with documented high restenosis 
[4]. Numerous studies provided sufficient evidence for a renewed class I recommendation by the European Society of Cardiology for in-stent restenosis with an evidence level A [5].

While initially safety and efficacy studies focused on ISR, de novo lesions were studied with paclitaxel-eluting stents as comparators. The BELLO study [6] randomized DCB and drug-eluting stents (DES) with paclitaxel coating to treat small-vessel de novo lesions. They found that late lumen loss was lower in the DCB group as compared to DES while the binary restenosis and revascularization rates were similar in both groups. Moreover, the clinical endpoint-powered BASKET SMALL II trial in small vessel de novo lesions [7] revealed comparable outcomes for DCB angioplasty and newer-generation DES. This growing clinical evidence supports the intuitive attractiveness of DCB angioplasty with its obvious benefit of avoiding coronary foreign body implants. The latter may also help to rationalize a shortened dual antiplatelet therapy (DAPT), in particular for elective patients with de novo lesions.

The existing body of evidence for the combination of CBA/SBA and DCB angioplasty, with the intention to avoid bailout stenting, was derived from heterogeneous clinical data. Previous study designs entailed either the use of different DCB devices [8] following lesion preparation with the same scoring balloon or different lesion preparation strategies [9] for one particular DCB device. Therefore, we set out to study the routine combination of a low-profile SBA with non-slip nylon elements $[9,10]$ and clinically proven iopromide-paclitaxel balloon angioplasty [11-14] in an unselected patient population with a focus on de novo coronary lesions.

\section{METHODS}

\section{Study Design and Population}

The PASSWORD study (Post MArket Surveillance of SeQuent Please NEO With ScORing Blesion Preparation in Real WorlD Patients) is a prospective, multicenter, single-armed study with the aim to document the safety and efficacy of a pre-specified SBA technology for routine lesion preparation in combination with iopromide-paclitaxel-coated balloon angioplasty (Paccocath ${ }^{\circledR}$ technology). The combination of the SBA/DCB lesion treatment strategy was intended to treat either in-stent restenosis of bare metal stents (BMS-ISR) or drug-eluting stents (DES-ISR) or de novo lesions with reference diameters between at least $2.5 \mathrm{~mm}$ and at most $4.0 \mathrm{~mm}$ and no greater than $30 \mathrm{~mm}$ in lesion length.

The main objective of the PASSWORD study was to assess the clinical suitability in terms of vessel patency and procedural success of the aforementioned combination treatment with a focus on stent-free de novo lesion treatment (ClinicalTrials.gov Identifier NCT02554292).

\section{Endpoints}

The primary endpoint was the rate of clinically driven target lesion failure (TLF) consisting of the rates for target lesion revascularization (TLR), myocardial infarction (MI), or cardiac death at 9 months. Cardiac death was defined as the cumulative rates of intra-hospital death of cardiac origin and all-cause death during follow-up.

TLR is defined as any repeat percutaneous intervention of the target lesion or bypass surgery of the target vessel performed for restenosis or other complication of the target lesion. Criteria for MI (intra-hospital and cumulative) were (a) the twofold increase of the upper normal value with significant proportion of creatine kinase (CK-MB), (b) the rise or fall in cardiac troponin (cTr) concentration with at least one value above the 99th percentile upper reference limit, or (c) greater than $20 \%$ rise in cTr [15]. As a result of the adherence to local clinical routines, electrocardiograms or cardiac enzymes were recommended prior to the intervention and before hospital discharge according to the aforementioned MI definition [15] and institutional guidelines of each participating center.

Secondary endpoints entailed the technical success rate to cross the target lesion and the individual rates for MI, TLR, cardiac mortality 
(in-hospital), and all-cause mortality (cumulated) in subgroups of de novo lesions, DES-ISR and BMS-ISR.

\section{Interventional Procedure}

The treatment recommendations of the German Consensus Group [16] for lesion preparation and DCB angioplasty were the basis for this study. All lesions were pre-dilated with the same type of SBA defined in this study with a balloon-to-vessel ratio of 0.8-1.0. Subsequent DCB angioplasty was only conducted in the absence of a major, flow-limiting dissection, i.e., less severe than type C-F according to the National Heart, Lung, and Blood Institute (NHLBI) classification and severe recoil at least 30\% of vessel diameter. An inflation time of at least $30 \mathrm{~s}$ at nominal pressures was recommended for DCB angioplasty whereas bailout stenting was permissible if severe dissections or residual diameter stenosis greater than 30\% occurred. Angiographic parameters were assessed and analyzed by clinical sites and the additional use of imaging modalities such as optical coherence tomography or intravascular ultrasound was left to the investigator according to his/her institutional guidelines.

\section{Statistics}

The $\chi^{2}$ test or the Fisher's exact test was used to detect differences between lesion subgroups when dichotomous variables were compared. The one-way analysis of variance was applied for continuous variables. As a result of the intended mélange of target lesions to be treated, a conservative TLF rate of $15.5 \%$ [17] was used as a reference while a TLF rate of $10.5 \%$ was assumed in the treatment group. On the basis of these rates, an alpha of $5 \%$, power of $80 \%$, and the null hypothesis $\mathrm{H}_{0}: \mathrm{TLF}_{\mathrm{DCB}+\mathrm{SBA}} \geq \mathrm{TLF}_{\text {historic, }}$ the sample size was estimated at 375 patients plus $20 \%$ to account for patients lost to followup. Hence, 450 patients were required. For all tests the significance level $\alpha$ was 0.05 . SPSS version 24.0 (IBM, Munich, Germany) was used for all analyses while the sample size was calculated with nQuery/nTerim Advisor, version 2.0 (Statistical Solutions Ltd. Cork, Ireland).

\section{Device Characteristics}

The scoring balloon used for lesion preparation (Fig. 1) consists of three non-slip scoring elements with triangular cross sections (Lacrosse NSE Nipro/Goodman, Nagoya, Japan/NSE Alpha B.Braun Melsungen AG, Germany). The device was used in one length only $(13 \mathrm{~mm})$.
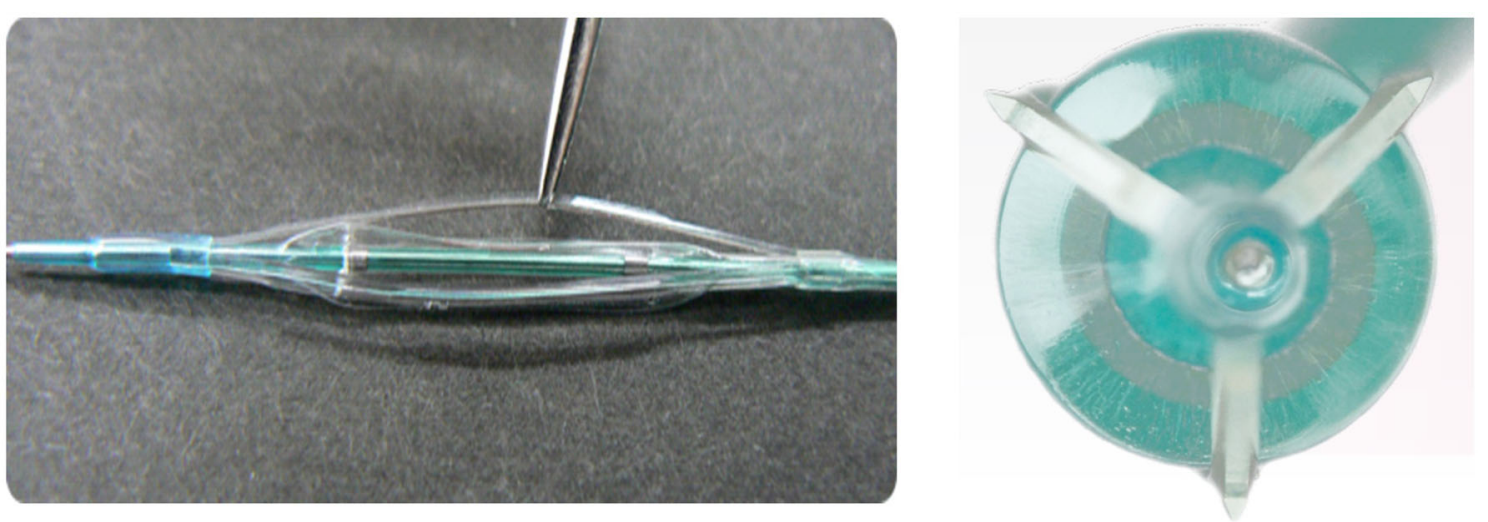

Fig. 1 Scoring balloon catheter with three nylon elements having a triangular cross section of height $0.39 \mathrm{~mm}$, bonded distally and proximally of the balloon (left panel, courtesy Nipro Corporation, Japan). When the balloon is inflated, the three non-slip elements are positioned on the cylindrical surface of the balloon spaced with $120^{\circ}$ from each other (right panel) 
Balloon diameters were available from 2.00 to $4.00 \mathrm{~mm}$. Calcified lesion modification with this device, as measured by optical coherence tomography, was favorably rated in previous observational studies [18]. Given the fact that there is no class effect for DCB devices [4] the iopromide-paclitaxel-coated DCB was used (SeQuent ${ }^{\circledR}$ Please NEO, B.Braun Melsungen $A G)$. As compared to the predecessor device (SeQuent ${ }^{\circledR}$ Please, B.Braun Melsungen AG), a hydrophilic coating of the distal shaft was introduced for enhanced lesion crossability.

\section{Co-medication}

Provided that patients were suitable for PCI, acetylsalicylic acid (ASA) 75-325 mg/day was recommended life-long. A clopidogrel loading dose of at least $600 \mathrm{mg}$, ASA $100 \mathrm{mg} /$ day orally 2 days pre intervention or $500 \mathrm{mg}$ ASA i.v. were recommended prior to the procedure. Alternative antiplatelet aggregation agents such as ticagrelor or prasugrel were also permissible at the discretion of the investigator. Intravenously administered heparin (70 IU/kg) or $5000 \mathrm{IU}$ was

Table 1 Patient demographics

\begin{tabular}{|c|c|c|c|c|c|}
\hline Variable & All & De novo & BMS-ISR & DES-ISR & $p$ value \\
\hline Number of patients & $481(100 \%)$ & $377(78.4 \%)$ & $19(4.0 \%)$ & $85(17.6 \%)$ & - \\
\hline Number of lesions & 496 & 386 & 19 & 91 & - \\
\hline Age $(\text { years })^{a}$ & $64.1 \pm 11.4$ & $62.8 \pm 11.2$ & $72.4 \pm 10.9$ & $68.1 \pm 10.8$ & $<0.001$ \\
\hline Male gender & $362(75.3 \%)$ & $289(76.7)$ & $16(84.2 \%)$ & $57(67.1 \%)$ & 0.117 \\
\hline Diabetes & $225(46.8 \%)$ & $167(44.3 \%)$ & $11(57.9 \%)$ & $47(55.3 \%)$ & 0.114 \\
\hline Hypertension & $394(81.9 \%)$ & $302(80.1 \%)$ & $18(94.7 \%)$ & $74(87.1 \%)$ & 0.108 \\
\hline Hyperlipidemia & $412(85.7 \%)$ & $317(84.1 \%)$ & $16(84.2 \%)$ & $79(92.9 \%)$ & 0.107 \\
\hline History of smoking & $257(53.4 \%)$ & $208(55.2 \%)$ & $9(47.4 \%)$ & $40(47.1 \%)$ & 0.345 \\
\hline Renal insufficiency & $116(24.1 \%)$ & $90(23.9 \%)$ & $7(36.8 \%)$ & $19(22.4 \%)$ & 0.399 \\
\hline Dialysis & $9(1.9 \%)$ & $6(1.6 \%)$ & $1(5.3 \%)$ & $2(2.4 \%)$ & 0.482 \\
\hline unstable angina & $112(23.3 \%)$ & $86(22.8 \%)$ & $3(15.8 \%)$ & $23(27.1 \%)$ & 0.516 \\
\hline \multicolumn{6}{|l|}{$\mathrm{AF}$} \\
\hline Paroxysmal & $22(4.6 \%)$ & $19(5.0 \%)$ & $1(5.3 \%)$ & $2(2.4 \%)$ & 0.466 \\
\hline Persistent & $6(1.2 \%)$ & $5(1.3 \%)$ & $0(0.0 \%)$ & $1(1.2 \%)$ & \\
\hline Long standing/persistent & $1(0.2 \%)$ & $1(0.3 \%)$ & $0(0.0 \%)$ & $0(0.0 \%)$ & \\
\hline Permanent & $12(2.5 \%)$ & $7(1.9 \%)$ & $2(10.5 \%)$ & $3(3.5 \%)$ & \\
\hline No AF & $440(91.5 \%)$ & $345(91.5 \%)$ & $17(84.2 \%)$ & $78(92.9 \%)$ & \\
\hline ACS & $99(20.6 \%)$ & $77(20.4 \%)$ & $6(31.6 \%)$ & $16(18.8 \%)$ & 0.456 \\
\hline STEMI & $20(4.2 \%)$ & $19(5.0 \%)$ & $1(5.3 \%)$ & $0(0.0 \%)$ & 0.161 \\
\hline NSTEMI & 79 (16.4\%) & $58(15.4 \%)$ & $5(26.3 \%)$ & $17(18.7 \%)$ & 0.161 \\
\hline
\end{tabular}

$A C S$ acute coronary syndrome, $A F$ atrial fibrillation, $B M S$-ISR in-stent restenotic lesions in bare metal stents, $D E S$-ISR instent restenotic lesions in drug-eluting stents, NSTEMI non-ST-elevation myocardial infarction, STEMI ST-elevation myocardial infarction

a Single-factor ANOVA, otherwise $\chi^{2}$ or Fisher's exact test whenever applicable 
recommended and adjusted when required according to each institution's guidelines.

\section{Data Collection}

An established electronic data capture system [11-14] was utilized to document baseline and follow-up data (www.studiesportal.com). The respective national principal investigators ensured the accuracy of the datasets whenever system integrated plausibility checks revealed inaccuracies, which initiated source data verification in the affected centers.

\section{Compliance with Ethics Guidelines}

The lead ethics committee of the coordinating investigator (Landesärztekammer Brandenburg No. AS 130/2015) first approved this study. Subsequently, pertinent ethics committees of all participating cardiac centers approved the study prior to patient recruitment. The declaration of Helsinki in its most recent form was the basis for this trial. Patients were informed and consented prior to enrollment.

\section{RESULTS}

\section{Patient Demographics}

A total of 481 patients were recruited for treatment of de novo lesions $(78.4 \%, 377)$, BMS-ISR $(4.0 \%, 19)$, and DES-ISR $(17.6 \%, 85)$. Overall risk factors were acute coronary syndrome (ACS, $20.6 \%, 99)$, diabetes mellitus $(46.8 \%, 225)$, and atrial fibrillation $(8.5 \%, 41)$ (Table 1$)$.

\section{LESION CHARACTERISTICS AND PROCEDURES}

The target vessel distribution (Table 2) between the three patient groups was not different $(p=0.492)$. Predominantly de novo lesions (77.8\%) followed by DES-ISR (18.4\%) were treated.

Average lesion lengths were $16.7 \pm 10.4 \mathrm{~mm}$ in the de novo group and $20.1 \pm 8.9 \mathrm{~mm}$ (BMS-
ISR) and $16.2 \pm 9.8 \mathrm{~mm}$ (DES-ISR) for the ISR groups. Bailout stenting in target lesions was done in $1.3 \%(5 / 386)$ of the de novo lesion subgroup.

Details on scoring balloon dimensions and DCB catheters characteristics are detailed in Table 3.

\section{DCB and Lesion Lengths}

On average, DCB balloon lengths were approximately $7.7 \mathrm{~mm}$ longer than the corresponding lesion lengths as compared to the $2.6 \mathrm{~mm}$ difference for BMS-ISR and the $6.5 \mathrm{~mm}$ difference for DES-ISR lesions. Sixty-five DCB catheters were of 35 or $40 \mathrm{~mm}$ length. These were used to treat long lesions $(33.4 \pm 18.1 \mathrm{~mm})$ whereas shorter DCBs $(\leq 30 \mathrm{~mm})$ were preferred for lesion lengths of $15.5 \pm 8.5 \mathrm{~mm}$.

The overall technical success rate to prepare the lesion with SBA and subsequent DCB angioplasty was high $(99.8 \%, 480 / 496)$.

\section{Co-medication}

Regarding the post-procedural dual-antiplatelet therapy (Table 4), clopidogrel was most frequently used $(70.9 \%, 341 / 481)$ followed by ticagrelor $(17.0 \%, 82 / 481)$ and prasugrel $(11.6 \%, 56 / 481)$. In stable coronary artery disease patients who did not receive bailout stents and/or did not have a remaining DAPT mandated by prior DES implantation, the DAPT duration was 4 weeks.

\section{Clinical Outcomes}

The follow-up rate relative to the primary endpoint was $96.3 \%$ (463/491) (Table 5). The overall accumulated TLF rate was 3.0\% (14/463) mostly driven by significantly higher target lesion revascularization rates in the BMS-ISR $(5.3 \%, 1 / 19)$ and the DES-ISR group $(6.0 \%$, 5/84). The Kaplan-Meier analysis (Fig. 2) revealed significant differences in TLF (log rank $p<0.001)$. There were no in-hospital events in any of the three patient subgroups. The accumulated TLF rates were significantly higher in the DES-ISR and the BMS-ISR groups as 
Table 2 Lesion characteristics

\begin{tabular}{|c|c|c|c|c|c|}
\hline Variable & All & De novo & BMS-ISR & DES-ISR & $p$ value \\
\hline Number of lesions & $496(100 \%)$ & $386(77.8 \%)$ & $19(3.8 \%)$ & $91(18.4 \%)$ & - \\
\hline \multicolumn{6}{|l|}{ Target vessel } \\
\hline LAD & $255(51.4 \%)$ & $192(49.7 \%)$ & $14(70.0 \%)$ & $49(53.8 \%)$ & \multirow[t]{6}{*}{0.492} \\
\hline $\mathrm{CX}$ & $111(22.4 \%)$ & $89(23.1 \%)$ & $2(10.5 \%)$ & $20(22.0 \%)$ & \\
\hline RCA & $116(23.4 \%)$ & $95(24.6 \%)$ & $3(15.8 \%)$ & $18(20.0 \%)$ & \\
\hline Graft & $6(1.2 \%)$ & $3(0.8 \%)$ & $0(0.0 \%)$ & $3(3.3 \%)$ & \\
\hline Ramus intermedius & $7(1.4 \%)$ & $6(1.6 \%)$ & $0(0.0 \%)$ & $1(1.1 \%)$ & \\
\hline Unknown & $1(0.2 \%)$ & $1(0.3 \%)$ & $0(0.0 \%)$ & $0(0.0 \%)$ & \\
\hline Total occlusion & $25(5.0 \%)$ & $19(4.9 \%)$ & $4(21.1 \%)$ & $2(2.2 \%)$ & 0.003 \\
\hline Chronic total occlusion & $15(3.0 \%)$ & $13(3.4 \%)$ & $1(5.3 \%)$ & $1(1.1 \%)$ & 0.443 \\
\hline Thrombus burden & $7(1.4 \%)$ & $5(1.3 \%)$ & $1(5.3 \%)$ & $1(1.1 \%)$ & 0.345 \\
\hline Diffuse vessel disease & $181(36.5 \%)$ & $157(40.7 \%)$ & $5(26.3 \%)$ & $19(20.9 \%)$ & 0.001 \\
\hline Calcification & $168(33.9 \%)$ & $128(33.2 \%)$ & $10(52.6 \%)$ & $30(33.0 \%)$ & 0.212 \\
\hline Ostial lesion & $67(13.5 \%)$ & $52(13.5 \%)$ & $1(5.3 \%)$ & $14(15.4 \%)$ & 0.502 \\
\hline Bifurcation lesion & $105(21.2 \%)$ & $101(26.2 \%)$ & $1(5.3 \%)$ & $3(3.3 \%)$ & $<0.001$ \\
\hline Severe tortuosity & $28(5.6 \%)$ & $26(6.7 \%)$ & $0(0.0 \%)$ & $2(2.2 \%)$ & 0.133 \\
\hline AHA/ACC type B2/C lesion & $180(36.3 \%)$ & $130(33.7 \%)$ & $13(66.8 \%)$ & $37(40.7 \%)$ & 0.006 \\
\hline \multicolumn{6}{|l|}{ Number of diseased vessels } \\
\hline Single & $171(35.6 \%)$ & $120(31.1 \%)$ & $4(21.1 \%)$ & $52(57.1 \%)$ & \multirow[t]{3}{*}{$<0.001$} \\
\hline Double & $216(44.9 \%)$ & $193(50.0 \%)$ & $7(36.8 \%)$ & $22(24.2 \%)$ & \\
\hline Triple & $94(19.5 \%)$ & $73(18.9 \%)$ & $9(42.1 \%)$ & $17(18.7 \%)$ & \\
\hline Reference diameter $(\mathrm{mm})^{\mathrm{a}}$ & $2.74 \pm 0.44$ & $2.63 \pm 0.37$ & $3.05 \pm 0.29$ & $3.15 \pm 0.42$ & $<0.001$ \\
\hline Lesion length ${ }^{\mathrm{a}}$ & $16.8 \pm 10.2$ & $16.7 \pm 10.4$ & $20.1 \pm 8.9$ & $16.2 \pm 9.8$ & 0.320 \\
\hline Degree of stenosis $(\%)^{a}$ & $84.9 \pm 10.0$ & $85.1 \pm 9.9$ & $83.2 \pm 9.9$ & $84.5 \pm 9.9$ & 0.630 \\
\hline \multicolumn{6}{|l|}{ TIMI flow prior to intervention } \\
\hline 0 & $25(5.0 \%)$ & $18(4.7 \%)$ & $421.1 \%)$ & $3(3.3 \%)$ & \multirow[t]{4}{*}{0.005} \\
\hline 1 & $16(3.2 \%)$ & $15(3.9 \%)$ & $0(0.0 \%)$ & $1(1.1 \%)$ & \\
\hline 2 & $168(33.9 \%)$ & $140(36.3 \%)$ & $3(15.8 \%)$ & $25(27.5 \%)$ & \\
\hline 3 & $287(57.9 \%)$ & $213(55.2 \%)$ & $12(63.1 \%)$ & $62(68.1 \%)$ & \\
\hline
\end{tabular}

AHA/ACC American College of Cardiology/American Heart Association, BMS-ISR in-stent restenotic lesions in bare metal stents, $C X$ left circumflex artery, DES-ISR in-stent restenotic lesions in drug-eluting stents, $L A D$ left anterior descending, $R C A$ right coronary artery, TIMI thrombolysis in myocardial infarction

a Single-factor ANOVA, otherwise $\chi^{2}$ or Fisher's Exact Test whenever applicable 
Table 3 Procedural data and device characteristics

\begin{tabular}{|c|c|c|c|c|c|}
\hline Variable & All & De novo & BMS-ISR & DES-ISR & $p$ value \\
\hline Number of lesions & 496 & 386 & 19 & 91 & - \\
\hline DCBs used & 528 & 410 & 21 & 97 & - \\
\hline Predilatation with scoring balloon & $496(100 \%)$ & $386(100 \%)$ & $19(100 \%)$ & $91(100 \%)$ & - \\
\hline Scoring balloon diameter $(\mathrm{mm})^{\mathrm{a}}$ & $2.53 \pm 0.45$ & $2.43 \pm 0.41$ & $2.71 \pm 0.31$ & $2.92 \pm 0.42$ & $<0.001$ \\
\hline Predilatation scoring balloon pressure $(\mathrm{atm})^{\mathrm{a}}$ & $11.6 \pm 3.3$ & $11.0 \pm 3.0$ & $15.4 \pm 3.9$ & $13.4 \pm 3.1$ & $<0.001$ \\
\hline $\mathrm{DCB}$ diameter $(\mathrm{mm})^{\mathrm{a}}$ & $2.71 \pm 0.45$ & $2.60 \pm 0.39$ & $3.00 \pm 0.35$ & $3.10 \pm 0.43$ & $<0.001$ \\
\hline DCB length $(\mathrm{mm})^{\mathrm{a}}$ & $24.1 \pm 7.1$ & $24.4 \pm 7.0$ & $22.7 \pm 7.0$ & $22.7 \pm 7.0$ & 0.070 \\
\hline $\mathrm{DCB}$ inflation pressure $(\mathrm{atm})^{\mathrm{a}}$ & $9.4 \pm 3.2$ & $8.7 \pm 2.6$ & $12.9 \pm 4.3$ & $11.5 \pm 3.9$ & $<0.001$ \\
\hline Bailout stenting in target lesion & $1.2 \%(6 / 496)$ & $1.3 \%(5 / 386)$ & $5.3 \%(1 / 19)$ & $0.0 \%(0 / 91)$ & 0.153 \\
\hline Additional DES in non-target lesions & $9(1.9 \%)$ & $6(1.6 \%)$ & $1(5.3 \%)$ & $2(2.4 \%)$ & 0.482 \\
\hline Overall technical success per patient & $480(99.8 \%)$ & $376(99.7 \%)$ & $19(100.0 \%)$ & $85(100.0 \%)$ & 0.871 \\
\hline Multi-vessel PCI & $184(38.3 \%)$ & $165(43.8 \%)$ & $8(42.1 \%)$ & $11(12.9 \%)$ & $<0.001$ \\
\hline Additional non-target lesions treated & $0.6 \pm 0.9$ & $0.8 \pm 1.0$ & $0.7 \pm 0.2$ & $0.5 \pm 0.1$ & $<0.001$ \\
\hline Type of DES-ISR & & & & & - \\
\hline PES-ISR & $1(0.9 \%)$ & $0(0.0 \%)$ & $0(0.0 \%)$ & $1(1.1 \%)$ & \\
\hline SES-ISR & $9(8.2 \%)$ & $0(0.0 \%)$ & $0(0.0 \%)$ & $9(9.9 \%)$ & \\
\hline ZES-ISR & $14(12.7 \%)$ & $0(0.0 \%)$ & $0(0.0 \%)$ & $14(15.4 \%)$ & \\
\hline BES-ISR & $4(3.6 \%)$ & $0(0.0 \%)$ & $0(0.0 \%)$ & $4(4.4 \%)$ & \\
\hline EES-ISR & $37(33.6 \%)$ & $0(0.0 \%)$ & $0(0.0 \%)$ & $37(40.7 \%)$ & \\
\hline Biomatrix-ISR & $11(10.0 \%)$ & $0(0.0 \%)$ & $0(0.0 \%)$ & $11(12.1 \%)$ & \\
\hline PF-SES-ISR & $5(4.5 \%)$ & $0(0.0 \%)$ & $0(0.0 \%)$ & $5(5.5 \%)$ & \\
\hline Absorb-ISR & $1(0.9 \%)$ & $0(0.0 \%)$ & $0(0.0 \%)$ & $1(1.1 \%)$ & \\
\hline Other DES-ISR & $7(6.4 \%)$ & $0(0.0 \%)$ & $0(0.0 \%)$ & $7(7.7 \%)$ & \\
\hline \multicolumn{6}{|l|}{ Type of BMS-ISR } \\
\hline CoCr-ISR & $6(5.5 \%)$ & $0(0.0 \%)$ & $6(31.1 \%)$ & $0(0.0 \%)$ & \\
\hline CrCo-ISR & $4(3.6 \%)$ & $0(0.0 \%)$ & $4(21.1 \%)$ & $0(0.0 \%)$ & \\
\hline Stainless steel ISR & $4(3.6 \%)$ & $0(0.0 \%)$ & $2(10.5 \%)$ & $2(2.2 \%)$ & \\
\hline Other BMS-ISR & $7(6.4 \%)$ & $0(0.0 \%)$ & $7(36.8 \%)$ & $0(0.0 \%)$ & \\
\hline Time since stenting, months ${ }^{\mathrm{a}}$ & $45.5 \pm 53.5$ & - & $127.1 \pm 60.3$ & $26.7 \pm 28.4$ & $<0.001$ \\
\hline
\end{tabular}

$B M S$ bare metal stents, $D C B$ drug-coated balloon, DES drug-eluting stent, EES everolimus-eluting stents, ISR in-stent restenosis, $P C I$ percutaneous coronary intervention, $P E S$ paclitaxel-eluting stent, $P F$ polymer-free, $S E S$ sirolimus-eluting stent, $Z E S$ zotarolimus-eluting stent

a Single-factor ANOVA, otherwise $\chi^{2}$ or Fisher's exact test whenever applicable 
Table 4 Peri-procedural drug therapy

\begin{tabular}{|c|c|c|c|c|c|c|}
\hline Drug type & Drug & All & De novo & BMS-ISR & DES-ISR & $p$ value \\
\hline Patients & & 481 & 377 & 19 & 85 & - \\
\hline \multirow[t]{9}{*}{ Pre PCI } & $600 \mathrm{mg}$ clopidogrel & $\begin{array}{l}195 \\
\quad(40.5 \%)\end{array}$ & $\begin{array}{l}158 \\
(41.9 \%)\end{array}$ & $6(31.6 \%)$ & $\begin{array}{l}31 \\
\quad(36.5 \%)\end{array}$ & $<0.001$ \\
\hline & $300 \mathrm{mg}$ clopidogrel & $46(9.6 \%)$ & $36(9.5 \%)$ & $2(10.5 \%)$ & $8(9.4 \%)$ & \\
\hline & 75 mg clopidgrel & $36(7.5 \%)$ & $30(8.0 \%)$ & $2(10.5 \%)$ & $4(4.7 \%)$ & \\
\hline & ASA only & $60(12.5 \%)$ & $34(9.0 \%)$ & $7(36.8 \%)$ & $\begin{array}{l}19 \\
(22.4 \%)\end{array}$ & \\
\hline & $180 \mathrm{mg}$ ticagrelor & $61(12.7 \%)$ & $50(13.3 \%)$ & $0(0.0 \%)$ & $\begin{array}{l}11 \\
(12.9 \%)\end{array}$ & \\
\hline & $90 \mathrm{mg}$ ticagrelor & $1(0.2 \%)$ & $0(0.0 \%)$ & $1(5.3 \%)$ & $0(0.0 \%)$ & \\
\hline & $60 \mathrm{mg}$ prasugrel & $45(9.4 \%)$ & $41(10.9 \%)$ & $1(5.3 \%)$ & $3(3.5 \%)$ & \\
\hline & $10 \mathrm{mg}$ prasugrel & $5(1.0 \%)$ & $5(1.3 \%)$ & $0(0.0 \%)$ & $0(0.0 \%)$ & \\
\hline & no preloading & $32(6.7 \%)$ & $23(6.1 \%)$ & $0(0.0 \%)$ & $9(10.6 \%)$ & \\
\hline \multirow[t]{4}{*}{$\begin{array}{l}\text { Post } \\
\text { PCI }\end{array}$} & Clopidogrel & $\begin{array}{l}341 \\
\quad(70.9 \%)\end{array}$ & $\begin{array}{l}268 \\
\quad(71.1 \%)\end{array}$ & $\begin{array}{l}16 \\
(84.2 \%)\end{array}$ & $\begin{array}{l}57 \\
(67.1 \%)\end{array}$ & 0.087 \\
\hline & Ticagrelor & $82(17.0 \%)$ & $58(15.4 \%)$ & $2(10.5 \%)$ & $\begin{array}{l}22 \\
\quad(25.9 \%)\end{array}$ & \\
\hline & Prasugrel & $56(11.6 \%)$ & $50(13.3 \%)$ & $1(5.3 \%)$ & $5(5.9 \%)$ & \\
\hline & Other & $2(0.4 \%)$ & $1(0.3 \%)$ & $0(0.0 \%)$ & $1(1.1 \%)$ & \\
\hline Triple therapy & $\begin{array}{l}\text { DAPT }+ \text { vitamin } K \text { antagonist or } \\
\text { NOAC }\end{array}$ & $7(1.5 \%)$ & $6(1.6 \%)$ & $0(0.0 \%)$ & $1(1.2 \%)$ & 0.829 \\
\hline
\end{tabular}

$A P T$ antiplatelet therapy, $A S A$ acetylsalicylic acid, $B M S$ bare metal stent, $D A P T$ dual antiplatelet therapy, $D E S$ drug-eluting stent, $I S R$ in-stent restenosis, $N O A C$ non-vitamin $\mathrm{K}$ oral anticoagulant, $P C I$ percutaneous coronary intervention

compared to the de novo subgroup $(p<0.001)$. Moreover, there was a considerable number of non-target vessel revascularizations (nTVR) in the overall cohort $(2.2 \%, 10 / 463)$. A subgroup analysis in patients treated for de novo lesions revealed that the TLF rates were $1.3 \%(4 / 313)$ in lesions treated with balloon lengths of at most $30 \mathrm{~mm}$ and $0.0 \%(0 / 46)$ in patients with balloon lengths greater than $30 \mathrm{~mm}$.

\section{De Novo Subgroup}

There were 119 patients with calcified de novo lesions and 241 patients without evidence of vessel calcification. Between these two groups, there were no differences in the TLF rates (calcified lesions $0.0 \% ; 0 / 119$ vs. non-calcified lesions $1.7 \%$; $4 / 237, p=0.158$ ). Likewise, there was no difference in TLF in lesions with reference diameters less than $3.0 \mathrm{~mm}$ and $3.0 \mathrm{~mm}$ or larger $(1.2 \% ; 3 / 260$ vs. $1.0 \% ; 1 / 100, p=0.901)$.

\section{DISCUSSION}

\section{Primary Endpoint}

The main angiographic benefit of stent implantations is the immediate luminal gain, 
Table 5 Clinical outcomes

\begin{tabular}{|c|c|c|c|c|c|}
\hline Variable & All & De novo & BMS-ISR & DES-ISR & $p$ value \\
\hline Number of patients & 481 & 377 & 19 & 85 & - \\
\hline Patients with clinical follow-up & $463(96.3 \%)$ & $360(95.5 \%)$ & $19(100.0 \%)$ & $84(98.8 \%)$ & 0.234 \\
\hline Time to discharge, days & $2.2 \pm 5.9$ & $2.0 \pm 6.3$ & $6.0 \pm 7.7$ & $2.0 \pm 2.1$ & 0.016 \\
\hline Follow-up time (months) ${ }^{\mathrm{a}}$ & $9.4 \pm 2.9$ & $9.5 \pm 2.9$ & $9.2 \pm 0.6$ & $9.3 \pm 3.2$ & 0.831 \\
\hline In hospital TLF & $0(0.0 \%)$ & $0(0.0 \%)$ & $0(0.0 \%)$ & $0(0.0 \%)$ & - \\
\hline In hospital Re-PTCA & $0(0.0 \%)$ & $0(0.0 \%)$ & $0(0.0 \%)$ & $0(0.0 \%)$ & - \\
\hline In hospital CABG & $0(0.0 \%)$ & $0(0.0 \%)$ & $0(0.0 \%)$ & $0(0.0 \%)$ & - \\
\hline In hospital MI & $0(0.0 \%)$ & $0(0.0 \%)$ & $0(0.0 \%)$ & $0(0.0 \%)$ & - \\
\hline In hospital cardiac death & $0(0.0 \%)$ & $0(0.0 \%)$ & $0(0.0 \%)$ & $0(0.0 \%)$ & - \\
\hline Accumulated TLF & $14(3.0 \%)$ & $4(1.1 \%)$ & $2(10.5 \%)$ & $8(9.5 \%)$ & $<0.001$ \\
\hline Accumulated TLR & $9(1.9 \%)$ & $3(0.8 \%)$ & $1(5.3 \%)$ & $5(6.0 \%)$ & 0.005 \\
\hline Accumulated Re-PTCA & $9(1.9 \%)$ & $3(0.8 \%)$ & $1(5.3 \%)$ & $5(6.0 \%)$ & 0.005 \\
\hline Accumulated CABG & $1(0.2 \%)$ & $0(0.0 \%)$ & $0(0.0 \%)$ & $1(1.2 \%)$ & 0.104 \\
\hline Accumulated MI & $2(0.4 \%)$ & $0(0.0 \%)$ & $0(0.0 \%)$ & $2(2.4 \%)$ & 0.011 \\
\hline Accumulated cardiac death & $4(0.9 \%)$ & $1(0.3 \%)$ & $1(5.3 \%)$ & $2(2.4 \%)$ & 0.018 \\
\hline Accumulated TVR & $11(2.4 \%)$ & $4(1.1 \%)$ & $1(5.3 \%)$ & $6(7.1 \%)$ & 0.003 \\
\hline Accumulated non-target vessel revascularization & $10(2.2 \%)$ & $5(1.4 \%)$ & $2(10.5 \%)$ & $3(3.6 \%)$ & 0.017 \\
\hline Accumulated vessel thrombosis & $1(0.2 \%)$ & $1(0.3 \%)$ & $0(0.0 \%)$ & $0(0.0 \%)$ & 0.866 \\
\hline
\end{tabular}

$B M S$ bare metal stent, $C A B G$ coronary artery bypass graft, $D E S$ drug-eluting stent, $I S R$ in-stent restenosis, $M I$ myocardial infarction, PTCA percutaneous transluminal coronary angioplasty, $T L F$ target lesion failure, TVR target vessel revascularization

a Single-factor ANOVA, otherwise $\chi^{2}$ or Fisher's exact test whenever applicable

which represents an instant gratification for the interventionalist. For the same reason, stentfree, DCB angioplasty does not provide stentlike results. Lesion preparation with SBA resulting in controlled dissections for luminal gain potentially enhances drug uptake in the vascular wall, albeit these data are currently not available. Within the framework of observational statistics, the results of our medium-sized cohort study are promising with low TLF rates in the de novo lesion group. The clinical outcomes compare well with prior studies in unselected patient populations. Wöhrle et al. [13] reported 9-month MACE rates in the 2.4-2.6\% range, which is in agreement with our rate of less than 2.0\%. The small vessel observational study by Zeymer and co-workers [14] revealed an accumulated MACE rate of $4.3 \%$. However, one needs to bear in mind that the purpose of this study was observational in nature and event underreporting may have occurred.

\section{Lesion Preparation and Plaque Modification}

Coronary lesions are usually associated with atherosclerotic plaques, which are frequently eccentric and heterogeneous in nature [19]. Unfortunately, plaque vulnerability, i.e., its 


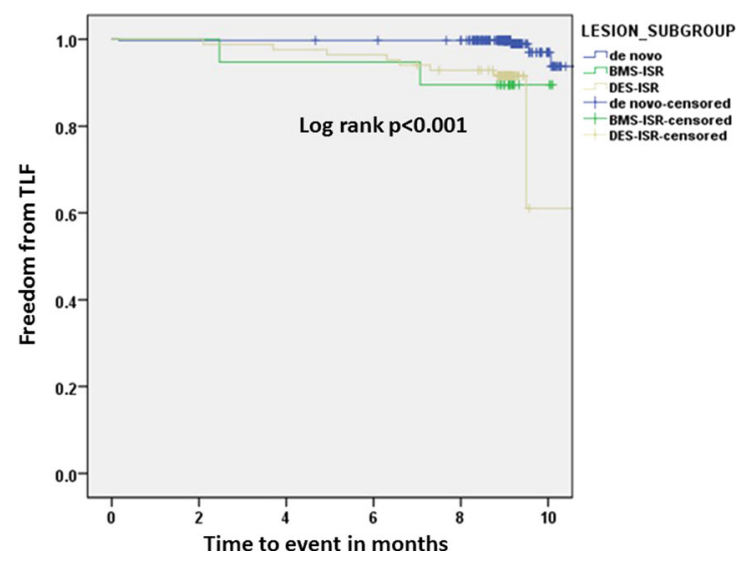

Fig. 2 Kaplan-Meier analysis for freedom from target lesion failure (TLF) with significant differences between lesion groups $(\log \operatorname{rank} p<0.001)$

potential to release the contents of encapsulated thrombogenic material, cannot be determined from fluoroscopic images [20]. However, a controlled plaque modification, i.e., a defined dissection mediated by the three nylon elements (Fig. 1) could be an option to obtain sufficient lumen gain and to reduce the risk of a post-procedural uncontrolled plaque rupture. Given the heterogeneity of the diseased coronary wall, it seems intuitive to use a mechanical cutting or scoring of this heterogeneous wall instead of a volumetric plaque displacement following balloon dilatation. Tzafriri et al. [21] studied local drug delivery after mechanical plaque modification in explanted human arteries. In calcified lower limb arteries, it was found that orbital atherectomy enhanced paclitaxel absorption and distribution in an ex vivo experimental setup. Despite the differences with atherectomy the non-slip nylon elements of SBA leading to an effective "cracking" in the vessel wall might have a similar effect to enhance drug transfer to the target cells. This combination of an improved acute lumen gain through SBA plus "paving the way" for local drug delivery through DCB angioplasty could explain the beneficial results of this single-arm registry.

\section{Bailout Stenting and Technical Success Rates}

It is noteworthy that bailout stenting was necessary in only $1.3 \%$ of de novo lesions. Only five lesions had dissections, which required extra intravascular scaffolding. An important finding in earlier studies is that despite the lower lumen gain at baseline, DCB angioplasty was shown to be associated with low TLR rates. If stents can be avoided, lumen enlargement over time, i.e., positive remodeling is possible [22]. DES even with latest-generation BMS backbones have stent thrombosis rates in the $0.9-1.9 \%$ range [23]. In our cohort, we recorded one vessel thrombosis, which did not occur in the target lesion.

In the de novo lesion group, key lesion morphological attributes were challenging with $33.2 \%$ calcification, $33.7 \%$ B2/C lesions, and $2.63 \pm 0.37 \mathrm{~mm}$ vessel diameters. Nevertheless, the technical success rate was $99.7 \%$ which is in agreement with the corresponding success rates of $99.2 \%$ from the Rosenberg registry $[11,12]$ and success rates from other registries ranging from $96.9 \%$ [13] to $98.9 \%$ [14].

\section{Scoring Before Drug Delivery}

There remains to be some debate whether a drug-coated scoring balloon may provide benefits relative to procedural and clinical outcomes. It is our conviction that scoring balloons ought to be the first step to check the feasibility of DCB angioplasty. In case of severe dissections, drug-coated SBA do not seem intuitive because of a potential "overmedication" in the target lesion, given the subsequent implantation of a DES.

\section{Final Remarks}

Based on the consensus group recommendations [16] for pre-dilating lesions prior to DCB angioplasty, the balloon-to-vessel ratio was defined in the range of 0.8-1.0. Even though it seems intuitive to reduce vessel segment trauma by not extending this ratio beyond 1.0, scientific evidence for this "sweet spot" is rooted in 
the early angioplasty work by Nichols et al. [24]. They found that the optimal balloon size in the beginning of balloon angioplasty in the range of 0.9-1.3 revealed the most favorable combination of minimal dissection risk and highest vessel patency. The very recent recommendations from the Asia-Pacific consensus group [25] engulfed two new aspects of lesion preparation and DCB angioplasty. Firstly, the authors found enough evidence to recommend DCB angioplasty also for de novo lesions in larger vessels supported by diagnostic techniques such as fractional flow reserve or optical coherence tomography. Secondly, another factor potentially contributing to vessel patency was introduced. The use of high dose statin [26] may significantly reduce plaque burden, thereby increasing coronary blood flow in the long term. One the other side, high dose statins may also modify plaque composition to render them more benign with less embolic potential.

\section{Limitations}

The dramatically changed regulatory environment requires real-world data in unselected patient populations with inherent data granularity. Despite the fact that our follow-up rate is high at greater than $95.0 \%$, event underreporting still remains a concern. However, event reporting by clinical sites was partly done via electronic health records, thereby reducing the likelihood of underreporting. Not all patients are suitable for DCB angioplasty and all efforts were made to reduce the selection bias. Nevertheless, only patients with successful lesion preparation following SBA angioplasty were subsequently treated with DCB angioplasty. Moreover, there is a significant learning curve and one has to assume that the procedural skills from study centers are not easily transferable to other cardiac centers. Despite the generally accepted recommendation for endpoint definition [27], we used the cumulative mortality rate instead of the cumulative cardiac death rate to pay tribute to the observational, clinical routine setting of this study which was mandated by regulatory agencies. A control angiography would have to be mandatory to meet this criterion. This type of follow-up, however, was outside of the scope of this clinical study.

\section{CONCLUSIONS}

Lesion preparation with SBA for DCB angioplasty is associated with low clinical event rates in particular for de novo lesions without additional stents. Our data suggest that especially patients undergoing stent-free DCB angioplasty may benefit the most from SBA-mediated plaque modification.

\section{ACKNOWLEDGEMENTS}

The authors wish to acknowledge the clinical research staff at the respective study sites as well as Denny Herberger (B. Braun Melsungen AG, Berlin, Germany) and Leigh Childs (Nipro/ Goodman, Nagoya, Japan) for their logistical support to conduct this study.

Funding. B. Braun Melsungen AG funded the rapid service fee, open access fee and the study with an unrestricted research grant. All authors had full access to all of the data in this study, contributed to the identification of the relevant literature, and were involved during all stages of the publication process to ensure the accuracy of the data analysis.

Authorship. All named authors meet the International Committee of Medical Journal Editors (ICMJE) criteria for the authorship of this publication. The responsibility to assure the integrity of this entire research work is equally shared by all authors who gave their approval to publish the finalized article.

Disclosures. Klaus Bonaventura is a consultant for B. Braun Melsungen AG. Matthias Waliszewski and Philip Steen are full-time employees (Medical Scientific Affairs, B. Braun Melsungen AG) when the manuscript was prepared. Markus Schwefer, Ahmad Khairuddin Mohamed Yusof, Florian Krackhardt, Raymundo Ocaranza, Ahmad Syadi Zuhdi, Liew 
Houng Bang, Kristof Graf, Ulrich Böck and Kenneth Chin have nothing to disclose.

Compliance with Ethics Guidelines. The lead ethics committee of the coordinating investigator (Landesärztekammer Brandenburg No. AS 130/2015) first approved this study. Prior to commencing this study, all relevant ethics votes were obtained in the participating countries. The declaration of Helsinki in its most recent form was the basis for this trial. Patients were informed and consented prior to enrollment.

Data Availability. The datasets generated during and/or analyzed during the current study are not publicly available but are available from the corresponding author on reasonable request.

Open Access. This article is licensed under a Creative Commons Attribution-NonCommercial 4.0 International License, which permits any non-commercial use, sharing, adaptation, distribution and reproduction in any medium or format, as long as you give appropriate credit to the original author(s) and the source, provide a link to the Creative Commons licence, and indicate if changes were made. The images or other third party material in this article are included in the article's Creative Commons licence, unless indicated otherwise in a credit line to the material. If material is not included in the article's Creative Commons licence and your intended use is not permitted by statutory regulation or exceeds the permitted use, you will need to obtain permission directly from the copyright holder. To view a copy of this licence, visit http://creativecommons.org/licenses/by$\mathrm{nc} / 4.0 /$.

\section{REFERENCES}

1. Mauri L, Bonan R, Weiner BH, et al. Cutting balloon angioplasty for the prevention of restenosis: results of the Cutting Balloon Global Randomized Trial. Am J Cardiol. 2002;90(10):1079-83.
2. Danek BA, Karatasakis A, Karacsonyi J, et al. A metaanalysis of contemporary lesion modification strategies during percutaneous coronary intervention in 244,795 patients from 22 studies. J Invasive Cardiol. 2017;29(12):E167-E176176.

3. Barbato E, Shlofmitz E, Milkas A, et al. State of the art: evolving concepts in the treatment of heavily calcified and undilatable coronary stenoses-from debulking to plaque modification, a 40-year-long journey. EuroIntervention. 2017;13(6):696-705. https://doi.org/10.4244/EIJ-D-17-00473.

4. Giacoppo D, Alfonso F, Xu B, et al. Paclitaxel-coated balloon angioplasty vs. drug-eluting stenting for the treatment of coronary in-stent restenosis: a comprehensive, collaborative, individual patient data meta-analysis of 10 randomized clinical trials (DAEDALUS study). Eur Heart J. 2019. https://doi. org/10.1093/eurheartj/ehz594.

5. Neumann FJ, Sousa-Uva M, Ahlsson A, et al. ESC Scientific Document Group. 2018 ESC/EACTS Guidelines on myocardial revascularization. Eur Heart J. 2019;40(2):87-165.

6. Latib A, Colombo A, Castriota F, et al. A randomized multicenter study comparing a paclitaxel drugeluting balloon with a paclitaxel-eluting stent in small coronary vessels: the BELLO (Balloon Elution and Late Loss Optimization) study. J Am Coll Cardiol. 2012;60(24):2473-80. https://doi.org/10.1016/ j.jacc.2012.09.020 (Erratum in: J Am Coll Cardiol. 2013 Apr 16;61(15):1660).

7. Jeger RV, Farah A, Ohlow MA, et al. BASKET-SMALL 2 Investigators. Drug-coated balloons for small coronary artery disease (BASKET-SMALL 2): an open-label randomised non-inferiority trial. Lancet. 2018;392(10150):849-56.

8. Merat B, Waliszewski M, Dillinger G, et al. Can you score with balloons to enhance outcomes after drug coated balloon angioplasty? Insights from the Paris DCB Registry for in-stent restenosis. J Interv Cardiol. 2018;31(3):353-9.

9. Yu X, Ji F, Xu F, et al. Treatment of large de novo coronary lesions with paclitaxel-coated balloon only: results from a Chinese institute. Clin Res Cardiol. 2019;108(3):234-43.

10. Ashida K, Hayase T, Shinmura T. Efficacy of lacrosse NSE using the "leopard-crawl" technique on severely calcified lesions. J Invasive Cardiol. 2013;25(10):555-64.

11. Rosenberg M, Waliszewski M, Chin $\mathrm{K}$, et al. Prospective, large-scale multicenter trial for the use of drug coated balloons (DCB) in coronary lesions: the DCB-only all-comers registry. Catheter 
Cardiovasc Interv. 2018. https://doi.org/10.1002/ ccd.27724.

12. Rosenberg R, Waliszewski W, Krackhardt F, et al. Drug coated balloon-only strategy in de novo lesions of large coronary vessels. J Interv Cardiol. 2019;2019:6548696. https://doi.org/10.1155/2019/ 6548696 (eCollection 2019).

13. Wöhrle J, Zadura M, Möbius-Winkler S, et al. SeQuent Please World Wide Registry clinical results of SeQuent Please paclitaxel-coated balloon angioplasty in a large-scale, prospective registry study. J Am Coll Cardiol. 2012;60(18):1733-8.

14. Zeymer U, Waliszewski $\mathrm{M}$, Spiecker $\mathrm{M}$, et al. Prospective 'real world' registry for the use of the 'PCB only' strategy in small vessel de novo lesions. Heart. 2014;100(4):311-6.

15. Thygesen K, Alpert JS, Jaffe AS, et al. Third universal definition of myocardial infarction. J Am Coll Cardiol. 2012;60:1581-98.

16. Kleber FX, Rittger H, Bonaventura K, et al. Drugcoated balloons for treatment of coronary artery disease: updated recommendations from a consensus group. Clin Res Cardiol. 2013;102(11):785-97.

17. $\mathrm{Xu} \mathrm{B}$, Gao R, Wang J, et al. A prospective, multicenter, randomized trial of paclitaxel-coated balloon versus paclitaxel-eluting stent for the treatment of drug-eluting stent in-stent restenosis: results from the PEPCAD China ISR trial. JACC Cardiovasc Interv. 2014;7(2):204-11.

18. Sugawara Y, Ueda T, Soeda T, et al. Plaque modification of severely calcified coronary lesions by scoring balloon angioplasty using Lacrosse non-slip element: insights from an optical coherence tomography evaluation. Cardiovasc Interv Ther. 2019;34(3):242-8.

19. Yamagishi M, Terashima M, Awano K, et al. Morphology of vulnerable coronary plaque: insights from follow-up of patients examined by intravascular ultrasound before an acute coronary syndrome. J Am Coll Cardiol. 2000;35(1):106-11.
20. Schoenhagen P, Nissen SE. Assessing coronary plaque burden and plaque vulnerability: atherosclerosis imaging with IVUS and emerging noninvasive modalities. Am Heart Hosp J. 2003;1(2):164-9.

21. Tzafriri AR, Garcia-Polite F, Zani B, et al. Calcified plaque modification alters local drug delivery in the treatment of peripheral atherosclerosis. J Control Release. 2017;28(264):203-10.

22. Kleber FX, Schulz A, Waliszewski M, et al. Local paclitaxel induces late lumen enlargement in coronary arteries after balloon angioplasty. Clin Res Cardiol. 2015;104(3):217-25. https://doi.org/10. 1007/s00392-014-0775-2.

23. Buiten RA, Ploumen EH, Zocca P, et al. Thin, very thin, or ultrathin strut biodegradable or durable polymer-coated drug-eluting stents: 3-year outcomes of BIO-RESORT. JACC Cardiovasc Interv. 2019;12(17):1650-60.

24. Nichols AB, Smith R, Berke AD, et al. Importance of balloon size in coronary angioplasty. J Am Coll Cardiol. 1989;13(5):1094-100.

25. Her AY, Shin ES, Bang LH, et al. Drug-coated balloon treatment in coronary artery disease: recommendations from an Asia-Pacific Consensus Group. Cardiol J. 2019. https://doi.org/10.5603/CJ.a2019. 0093.

26. Hwang DS, Shin ES, Kim SJ, et al. Early differential changes in coronary plaque composition according to plaque stability following statin initiation in acute coronary syndrome: classification and analysis by intravascular ultrasound-virtual histology. Yonsei Med J. 2013;54(2):336-44. https://doi.org/ 10.3349/ymj.2013.54.2.336.

27. Garcia-Garcia HM, McFadden EP, Farb A, et al. Academic Research Consortium standardized end point definitions for coronary intervention trials: the Academic Research Consortium-2 consensus document. Eur Heart J. 2018;39(23):2192-207. 